Word Count: 6000

Number of Tables: 2

Number of Figures: 0

Online supplemental tables: 2

Online supplemental figures: 1

Online Appendices: 2

Number of References: 65

\title{
A systematic review of psychotherapeutic interventions for women with metastatic breast cancer: Context matters.
}

Lisa Beatty ${ }^{1,2}$, Emma Kemp ${ }^{1,2}$, Phyllis Butow ${ }^{3}$, Afaf Girgis ${ }^{4}$, Penelope Schofield ${ }^{5-7}$, Jane Turner $^{8}$, Nicholas J. Hulbert-Williams ${ }^{9}$, Janelle V. Levesque ${ }^{4}$, and Bogda Koczwara ${ }^{2}$

${ }^{1}$ Flinders University, Adelaide, SA, 5001, AUSTRALIA

${ }^{2}$ Flinders Centre for Innovation in Cancer, Bedford Park, SA, 5042, AUSTRALIA

${ }^{3}$ University of Sydney, Sydney, NSW, AUSTRALIA

${ }^{4}$ Ingham Institute for Applied Medical Research; South Western Sydney Clinical School, University of New South Wales, Sydney, NSW, AUSTRALIA

${ }^{5}$ Department of Psychology, School of Health Sciences, Faculty of Health, Arts and Design, Swinburne University of Technology, Hawthorn, Australia

${ }^{6}$ Department of Cancer Experiences Research, Peter MacCallum Cancer Center, East Melbourne, VIC, Australia

${ }^{7}$ Sir Peter MacCallum Department of Oncology, Faculty of Medicine, Dentistry and Health Sciences, The University of Melbourne, Parkville, VIC, Australia

${ }^{8}$ University of Queensland, Brisbane, NSW, Australia

${ }^{9}$ Chester Research Unit for the Psychology of Health, University of Chester, Chester, UNITED KINGDOM

This is the author manuscript accepted for publication and has undergone full peer review but has not been through the copyediting, typesetting, pagination and proofreading process, which may lead to differences between this version and the Version of Record. Please cite this article as doi: $10.1002 /$ pon.4445

This article is protected by copyright. All rights reserved. 
Corresponding Author: Dr Lisa Beatty, School of Psychology, Flinders University, GPO Box 2100, Adelaide, SA, 5001, Australia. TEL: +61 88201 2506; FAX: +61 88201 3877; email: lisa.beatty@flinders.edu.au

\begin{abstract}
Objectives: To summarise the evidence-base of psychological interventions for women with metastatic breast cancer (MBC), by mode of delivery (group, individual, or low-intensity interventions). To synthesise data regarding core intervention-elements (e.g., intervention duration) and context factors (trial setting, uptake and adherence, demographic characteristics).

Methods: Four databases were searched (inception - May 2016): MEDLINE (OvidSP), PsycINFO (OvidSP), CINAHL (EBSCO), and SCOPUS; reference lists were examined for additional publications. Grey literature was excluded. Outcome data were extracted for survival, distress, quality of life, coping, sleep, fatigue, and/or pain, and summarised through narrative synthesis.
\end{abstract}

Results: Fifteen randomised clinical trials (RCTs), reported across 23 articles, met inclusion criteria: seven group, four individual, and four low-intensity interventions. Overall, interventions improved distress (8/13 RCTs); coping (4/5 RCTs); and pain (4/5 RCTs). No evidence of survival benefit was found. For remaining outcomes, evidence was either insufficient, or too mixed to draw conclusions. Group programs had the strongest evidencebase for efficacy; individual and low-intensity therapy had insufficient evidence to form conclusions. Group interventions had longest intervention durations and lowest uptake and adherence; low-intensity interventions had shortest durations and highest uptake and adherence. Disparities in uptake, adherence and reach were evident, with the demographic profile of participants polarised to young, Caucasian, English-speaking, partnered women.

Conclusions: There remains a paucity of psychological interventions for women with MBC. Those that exist have an inconsistent evidence-base across the range of patient-reported outcomes. Further research is needed to evaluate accessible delivery formats that ensure efficacy as well as uptake.

\title{
Abstract word count: 245
}

Keywords: cancer, oncology, metastatic breast cancer, psychological interventions, systematic review, treatment-modality 
Metastatic breast cancer (MBC) is defined as cancer that has spread from the breast to more distant parts of the body, most commonly the bones, lungs, liver, and brain [1]. Despite the advent of new systemic therapies, the median survival remains approximately two to three years [2], although some women experience extended survival periods of over 10 years.

A diagnosis of advanced breast cancer brings significant adjustment challenges for women, as they face a future of physical symptoms, functional limitations, and existential concerns about their ultimate mortality. Clinically elevated distress and impaired health-related quality of life (HRQOL) occur in 35-43\% [3] of women following diagnosis of MBC. Given that psychological factors, particularly depression, have been implicated in reduced survival time [4], increased severity of symptoms[5], and reduced treatment adherence [6], psychosocial interventions for this population are warranted.

However, contrasting to the 28 randomised controlled trials (RCTs) of psychological interventions for 3940 participants with early-stage breast cancer [7], the Cochrane Collaboration found only 10 RCTs had been specifically conducted for 1378 women with MBC [8]. This MBC Cochrane Review, a meta-analysis published in 2013, included papers published between 1989 and 2010, with seven of the 10 included RCTs evaluating a grouptherapy program of either a supportive-expressive $(n=4)$, or cognitive behavioural $(n=3)$ framework [8]. The remaining three studies trialled individual face-to-face therapies, and did not utilise either of these therapeutic frameworks. Overall, the Cochrane Review found a short-term psychological benefit of MBC-interventions for improving pain and psychological outcomes (distress, helplessness/hopelessness, social functioning, cancer-related distress, emotional control), and a modest survival benefit at one year which was not sustained at five years [8].

Since publication of the Cochrane Review, there has been a surge of interest in the provision of online self-directed psychological interventions targeting illness-related distress [9-18]. Furthermore, recent survey data indicates that psychosocial needs remain the key concern of women with MBC, with self-care strategies being listed as the most-preferred or secondpreferred option for distress management [19]. These web-based and self-care strategies form part of an emerging modality for receiving psychological interventions, termed 'lowintensity' [20]. The primary purpose of low intensity interventions is to increase access to evidence-based psychological therapies, using the minimum level of intervention necessary to create maximum gain, and delivering content in a variety of flexible forms, including email, smart-devices, telephone, print-based resources, and internet [20]. While definitions vary, the key attributes of low-intensity interventions are that they are more accessible; use fewer formal healthcare professionals' resources compared to conventional therapies; and may use non-specialist practitioners to deliver the programs [20,21]. In contrast, group and individual psychotherapeutic interventions are high-intensity, defined as therapistadministered programs, delivered in-person by health professionals with specialist training [8], and can be of either brief (less than 6 hours) or extensive duration [21]. Considering the research and patient preference developments relating to low-intensity interventions, particularly web-based psychological therapy, it is timely to investigate whether they have 
penetrated the MBC-literature, and to update the evidence in light of any new, or previously missed, trials that have been published.

Given the variety of formats now available for disseminating psychological interventions, it is important to evaluate how the core elements of an intervention (modality of delivery, and their differing treatment durations) impact on outcomes. While the prior Cochrane Review summarised the number of group and individual interventions included, they did not compare outcomes by delivery modality [8]. Furthermore, it is also becoming increasingly recognised that research data must be considered within the context they were obtained. That is, to identify (a) in what setting, (b) under what conditions, and (c) for which specific section of the population the evidence was derived, in order to then understand the implications for subsequent implementation. These context and intervention-elements data have not previously been summarised, but have important implications for reach and uptake.

This current review therefore aimed to (a) update efficacy findings from a previous Cochrane Review of psychological interventions for women with MBC, to include any missed RCTs, or RCTs published in the subsequent 5 years, (b) summarise outcomes by treatmentmodality, and (c) synthesise the following intervention-duration and trial context data: rates of uptake (defined as the number of consenting participants as a proportion of approached eligible individuals, e.g. [22]), adherence (the amount of an intervention an individual engages with or completes [23]), the geographic and medical settings of trials conducted, and the demographic profile of included participants.

\section{Method}

\section{Methodological Framework}

The Preferred Reporting Items for Systematic Reviews and Meta-Analyses (PRISMA) statement was used [24] and a review protocol was developed (Online Appendix 1).

\section{Data Sources}

Two methods of identifying primary studies were utilised. First, one author (LB) conducted four electronic bibliographic database searches (from each database's inception to May 2016): MEDLINE, PsycInfo, CINAHL and SCOPUS. A list of keywords and MeSH terms were generated to identify studies, falling within three key search strategies (for full details, see online supplemental materials Appendix 1 - Review protocol):

Terms related to metastatic breast cancer: 'metasta*', 'secondary', 'advanced', 'breast cancer';

Terms related to psychological intervention: 'psycho*', 'intervention', 'psychotherapy', 'group support*', 'self-help' 'internet*', 'tele-counsel*'; Terms related to outcomes: 'survival'; 'quality of life', 'psychosocial'.

This initial search identified 1151 citations (740 after de-duplication). The reference lists of the included primary studies and previous systematic reviews were then screened to identify further articles. The full electronic search strategy used for one database (PsycINFO) is 
included in Online Appendix 2. Figure 1 (see online supplemental materials) summarises the search and retrieval process utilised.

\section{Study Selection}

The title and abstract of each citation were examined against the following pre-specified inclusion and exclusion criteria:

1. The article was published, or in-press, in a peer-reviewed journal between database inception and $25^{\text {th }}$ May 2016. Grey literature was excluded.

2. The intervention targeted adult women with a diagnosis of metastatic or recurrent breast cancer.

3. Studies including women with $\mathrm{MBC}$ as part of a larger sample of patients with other cancer types or stages were eligible for inclusion if data could be extracted specific to women with MBC. There were no restrictions on the minimum MBC-sample size required.

4. The intervention was psychotherapeutic in nature (i.e., provided psychological strategies, of any therapeutic framework, to manage illness-related challenges). Interventions providing information/education without a therapeutic component were excluded.

5. Survival, distress, coping, quality of life, or somatic symptoms was an outcome.

6. The study was a randomised controlled trial (RCT), quasi-randomised trial, or feasibility RCT.

7. The article was published in English.

Initial assessment of eligibility was performed by EK based on titles and abstracts. Database searches were then replicated by one other author (LB or BK). There was $97.6 \%$ agreement $($ kappa $=0.767)$ on the selected review articles. Disagreement in selection was resolved through discussion between authors. Full-texts of citations meeting initial inclusion criteria were obtained. All full-texts were independently examined by two authors (LB and EK) to confirm inclusion/exclusion, with $100 \%$ agreement obtained.

\section{Data Extraction}

Data were extracted independently by two members of the authorship group using a standardised coding sheet, based on the Cochrane library's recommendations for data extraction [25]. Where discrepancies occurred in the data extracted by the initial reviewing authors, a third author (LB/EK) reviewed the data and consensus was reached through discussion. Interventions were coded as either 'Group', 'Individual', or 'Low-Intensity' during extraction and synthesis. Group and individual interventions were stated as such in papers and were delivered in person. To be classified as 'Low-Intensity', interventions had to meet four criteria drawn from definitions of low-intensity therapies [20, 21]: (a) administered in a non-face-to-face setting; (b) delivered by non-mental health practitioners; (c) be more accessible; and (d) be briefer for the practitioner compared to therapist-delivered treatments..

\section{Quality Assessment}


The quality of each paper was assessed by two authors independently: the research design quality was assessed using the five criteria for empirically supported psychotherapies [26]; and the quality of reporting was secondly assessed at the study level using six criteria from the Cochrane Collaboration's 'risk of bias' tool [25].

\section{Data Synthesis}

A narrative synthesis of results was utilised due to the substantial heterogeneity in study design, population, type of intervention, outcomes, and timing of assessments. The first author synthesised results, with findings verified by the authorship group.

\section{Results}

\section{Flow of studies through the review}

From the initial pool of 740 screened titles and abstracts, 47 citations met inclusion criteria for full review. Two potential studies were identified from reference lists of the initial fulltexts screen. Of these 49 total citations, 26 were subsequently excluded during full-text analysis, because: MBC-specific patient data could not be extracted from larger samples of mixed cancer types $(n=14)$; the study was not an RCT $(n=6)$; the intervention was not psychotherapeutic $(n=4)$; or the publication did not evaluate efficacy $(n=2)$. Therefore 23 articles (from 15 RCTs) were included [27-49]. Five RCTs not summarised by the previous Cochrane review were identified and included in this review; four were published prior to the previous Cochrane Review update [28, 37, 41, 48]. Of note, only one new RCT was published in the past 5 years [43].

\section{Overview of included studies}

Table A (see online supplemental materials) summarises the characteristics of the 15 RCTs reported across 23 articles, with studies listed by treatment-delivery modality. A total of 1638 women participated in the RCTs; seven interventions (50\%) were delivered in a group format [31-33, 38, 40, 41, 45]; four in individual therapy [27, 28, 44, 48]; and four as 'low-intensity' (telephone counselling, $n=2$; expressive writing, $n=2$ ) [37, 39, 42, 43]. None of the included studies trialled online/web-based therapy. Most $(n=13)$ interventions fell into five therapeutic frameworks: (i) Supportive Expressive Group Therapy (SEGT; n=4)[31, 38, 40, 45]; (ii) Cognitive Behaviour Therapy (CBT; $n=5)$ [28, 33, 37, 44, 48]; (iii) SEGT/CBT combined $(\mathrm{n}=1)$ [32]; (iv) Expressive Writing ( $\mathrm{n}=2$ ) [42, 43]; (v) Hypnosis [41]; with the remaining two RCTs trialling telephone counselling [39], and a nurse-lead counselling program [27]. Program duration ranged from 80 minutes (expressive writing), to 78 hours (SEGT); treatment providers ranged from none (expressive writing) to two highly skilled co-therapists (CBT, SEGT); and the setting was predominantly urban hospitals or clinics $(n=11 / 15)$.

\section{Methodological quality}


Table B (see online supplemental materials) summarises the methodological quality of the included RCTs. Overall, the methodological quality was moderate. While no RCT met all 11 combined criteria, eight (53\%) RCTs met at least eight of the 11 combined criteria [31-33, $38-40,43,48]$. The majority of these higher quality studies were group-delivered [31-33, 38, 40]; one individual therapy [48], and two low-intensity [39, 43] studies met this quality criteria threshold.

Looking at the criteria separately, six trials (40\%) met all the research design criteria [31, 33, 38-40, 43]. All trials utilised acceptable control conditions: eight RCTs utilised less methodologically rigorous comparators (usual care, $n=5$ [27, 28, 33, 39, 45]; waitlist control, $\mathrm{n}=3[41,44,48]$ ); while seven had stronger control conditions (attention-control, $\mathrm{n}=5[31,38$, 40, 42, 43]; active-treatment control, $n=2$ [32, 37]). Most studies $(n=11)$ had sample sizes larger than 25 participants per group, however only six trials reported their power calculations and were sufficiently powered to detect moderate effects. All except one study [44] specified inclusion criteria, and only two studies did not clearly report or did not use reliable/well-validated measures [37, 44].

No RCT met all 6 reporting criteria for risk of bias [25]. Twelve studies (80\%) gave sufficient information about how the allocation sequence was generated, and nine of these gave sufficient information about whether allocation was adequately concealed. Only two studies specifically met the criteria for blinded assessment by research personnel (no trial reported on participant blinding) [43, 48]. Strategies to manage incomplete outcome data were reported in seven (47\%) RCTs, while nine trials were free of selective reporting of outcomes. Finally, only five RCTs $(33 \%)$ were clearly free of other bias, with the majority of studies being either unclear in their reporting $(n=7)$ or not meeting this criterion $(n=3)$.

\section{Trial Outcomes}

There was marked heterogeneity in outcomes, measures utilised, and timing of assessments in included trials. Only seven RCTs specified a primary outcome (group: $n=4$, individual: $n=1$, low-intensity: $n=2)$; specifically, survival $(n=3)$; mood disturbance $(n=2)$; quality of life $(\mathrm{n}=1)$, and symptom severity response time $(\mathrm{n}=1)$. In order to increase clarity of findings, this review consolidated outcomes into the following domains: (a) survival; (b) distress (incorporating depression, anxiety, stress, cancer-related distress, intrusive thoughts); (c) coping (incorporating mental adjustment domains such as helplessness, denial, repression); (d) quality of life; and the commonly reported cancer symptoms of (e) pain (intensity, suffering, sensation, duration, perceived ability to control); (f) fatigue; and (g) sleeping difficulties. Given the marked heterogeneity of number and timing of assessments, and the analyses conducted, comparing the short versus long term benefits of interventions was not possible. This review therefore adopted an inclusive criterion coding an intervention as a positive trial if it yielded a favourable impact on an outcome for at least one of the follow-up assessments. Table 1 summarises the evidence-base per outcome domain, by treatmentdelivery modality for the included trials, with an overall (pooled) summary of the evidencebase. This review did not synthesise outcomes analysed in fewer than two trials: these 
included social support, self-efficacy, and self-esteem; these outcomes were instead reported per individual study in Table A (see online supplemental materials).

\section{Survival}

Six RCTs investigated survival as an outcome, all delivered as group programs $[32,34,38$, 40, 46, 47]; only the first published study [47] found a statistically significant survival benefit resulting from group participation. Of note, none of the three studies that were specifically powered for survival as a primary outcome obtained significant effects $[32,38,40]$.

\section{Distress}

Pooling across intervention types, 'distress' was the most frequently assessed outcome, investigated in 13 trials [28, 31-33, 37-43, 45, 48]: eight RCTs demonstrated efficacy for at least one distress outcome $[31,33,37,38,40,41,45,48]$, of which seven tested established therapeutic frameworks (i.e., SEGT, CBT). Breaking results down by treatment modality, Table 1 demonstrates that six of the seven group-therapy trials examining distress were positive (86\%), including the single trial that specified distress as a primary outcome [31]; one of the two individual therapy trials was positive (50\%), while one low-intensity trial obtained significant effects $(25 \%)$, with the remaining three low-intensity RCTs, including one that specified distress as a primary outcome [39], failing to find effects. Therefore, while the evidence-base for psychological therapy (pooled across treatment modalities) for distress was supported, only group-delivered programs consistently demonstrated positive results.

\section{Coping}

Five trials examined coping domains as an outcome [31, 32, 40, 44, 45]. Four were groupdelivered; and all four obtained positive effects, therefore reaching criteria for being considered empirically supported. However, findings were significant only for certain domains; helplessness/hopelessness [32, 40], restraint [31], and a 'maladaptive coping' subscale [45], but not for other commonly-reported coping styles including denial, defensiverepressive coping, anxious preoccupation, cognitive avoidance, fatalism or fighting spirit [31, $32,40,45]$. The fifth trial was for individually-administered therapy [44], where coping was not statistically significantly impacted by the intervention. No trial to date has examined the impact of low-intensity interventions on coping outcomes.

\section{Quality of Life}

Six RCTs evaluated quality of life: three were group programs [32, 38, 40]; three were individual therapy $[27,44,48]$. Only one group-therapy trial reported statistically significant effects [40], in the specific QOL domain of social functioning, with the remaining five RCTs failing to find an effect, including one that specified QOL as a primary outcome [44]. Psychological interventions for quality of life are therefore currently unsupported, both within delivery-modalities and overall.

\section{Pain}


Pain was evaluated in five RCTs: three group trials [29, 30, 49]; one individual [28], and one low-intensity [37]. When pooling across the RCTs, four of the five psychological interventions trialled had an impact on pain intensity, sensation, suffering or perceived control over pain [28-30, 49]. Only the low-intensity intervention did not have an impact on pain [37]. Overall the use of psychological interventions, delivered in a face-to-face format (either group or individually), is currently supported; however the lack of trials for individual and low-intensity modalities preclude definitive recommendations being formed.

\section{Fatigue}

Fatigue was evaluated in five RCTs: two group interventions [29, 45]; one individual therapy [48]; and two low-intensity interventions [37, 43]. Only one of the five trials, a group intervention [45], demonstrated efficacy. Based on the current evidence, there is insufficient data to draw conclusions regarding the use of psychological intervention to treat cancerrelated fatigue in MBC.

\section{Sleeping Difficulties}

Four trials examined sleep: one individual intervention [48], and three low-intensity [37, 42, 43]. Two of the three low-intensity RCTs found evidence of benefit [37, 42]; however, for one of these trials, the benefits only applied to those 'recently' diagnosed with MBC, defined as within the past 20 months [42]. The remaining two trials failed to obtain significant results for improving sleep. The impact of psychological interventions, both overall or by treatmentmodality, on sleep-related difficulties therefore remains unclear based on the limited data currently available.

\section{Trial Context}

Table 2 summarises intervention duration and contextual factors associated with trial participation. As can be seen, the overall number of participants and average sample size per trial differed between treatment modalities: Group and low intensity intervention trials had comparably large sample sizes, with an average sample size per trial of 128 and 129.5 women respectively, in contrast to the average trial sample size of 54 women for RCTs of individually-administered treatments.

\section{Participant characteristics}

As Table 2 indicates, participant characteristics were not reported uniformly. Of the available data, the majority of studies included women who were young (mean age $=53.08$ years), less than two years post-diagnosis of MBC at study entry (pooled time since diagnosis $=1.96$ years), partnered/married (68.4\% of included study participants), English speaking (86.7\% of studies), and Caucasian (89.8\% of included studies). While $62.2 \%$ of participants overall were tertiary educated, only $31.9 \%$ of participants were employed. While participant characteristics in general did not differ between treatment modalities, a notable exception was education level: based on the eight studies reporting education, only $47.3 \%$ of group participants were tertiary educated, compared to $58.5 \%$ and $80.9 \%$ of individual and low- 
intensity participants, respectively. Group participants were nearly five years younger than those in individual therapy, and three years younger than low-intensity intervention participants, while women in individual therapy were more recently diagnosed than the other two modalities.

\section{Intervention characteristics}

As Table 2 summarises, group interventions had the highest participationburden/commitment, as measured by the maximum number of hours of treatment a participant might receive. Group interventions had an average intervention duration of 60 hours (ranging from 16 to 78 hours). This was notably higher than in individually-delivered (6.83 hours), and low intensity (3.79 hours) interventions, and nearly double the pooled interventions' average-duration of 32.6 hours.

Only 10 trials [27, 32, 33, 38, 40-43, 45, 48] summarised uptake data, while all 15 trials reported on adherence. As the number of intervention-hours decreased, the uptake rate increased: uptake was highest at $71.9 \%$ amongst low-intensity interventions (range 69-89\%) and lowest at $41.9 \%$ for group delivery formats (range 19-79\%), with individuallyadministered therapy falling in between (60.5\%; range 60-61\%). As Table 4 demonstrates, a similar pattern was observed for adherence, with the exception that individual therapy had lower adherence than group programs.

\section{Discussion}

Since 1980, only 15 trials (published across 23 articles) using rigorous randomised controlled designs, have examined psychotherapeutic interventions for MBC. Only one new study has been published in this area in the last five years and no studies of web-based interventions have been published. This was contrary to expectations, given that research and service provision for women with MBC has increasingly been recognised as a priority area [19], combined with the interest in web-based interventions for cancer [9-16].

No evidence of a survival benefit was found in the present review; while this contrasts with the one-year survival benefit found in the earlier Cochrane Review [8], the present review examined overall survival, rather than separating 1-year versus 5-year survival rates, and was therefore consistent with the Cochrane Review finding of no survival-benefit at five years. This finding was unsurprising. given that only one additional RCT was identified which examined this relationship [44]. This study had a very small sample size $(n=43)$, did not have survival as its primary aim, and was underpowered for such an analysis; furthermore, the final assessment period was only six months after study entry, therefore too short to examine survival advantage adequately. While the mechanism for increasing survival is plausible, given the postulated relationship between depression and reduced survival $[4,50]$; findings indicating stress may lead to cancer progression [51]; that social support/marital status can have as powerful an impact on survival as chemotherapy [52]; and that depression reduces adherence to medical treatments [53], to date none of the interventions trialled appear to have yielded large enough effects to impact on these mechanisms. A trial examining whether treating depression among those most vulnerable - such as socially isolated individuals - can 
improve survival would be beneficial. While promising, such a study would be difficult to power and conduct, given the large sample size and very long-term follow-up required.

Consistent with earlier reviews, this review did obtain evidence to support the use of psychological therapies overall (pooling across delivery-modality) for distress, select coping domains (helplessness/hopelessness and restraint-coping), and pain. This positive impact on distress is particularly notable, given that for all included studies, except one [48], elevated distress was not an inclusion criteria, therefore floor effects may have been operating. For the remaining psychosocial outcomes, namely quality of life, fatigue, and sleep, the evidence was either insufficient, or too mixed to draw conclusions, indicating that further research on the use of psychological therapies to improve these outcomes in women with MBC is warranted.

When summarising outcomes by treatment modality, this study indicates that the evidencebase for psychological therapies has been largely generated by group therapy studies, which were the most intensive (i.e., weekly for up to a year). Individual interventions did not meet criteria for a supported intervention for any outcome; however, as most outcomes were only investigated in single studies, further research in this area is required to clarify findings. Only tentative support was found for low-intensity interventions regarding one outcome, sleep; no support was found for fatigue and distress, and an unclear evidence-base was noted for pain. Therefore, at this stage both individual and low-intensity interventions have insufficient evidence to form conclusions regarding efficacy or to make recommendations for their use, and it remains premature to directly compare group interventions with other modalities of support. While groups were most efficacious, the program efficacy must be balanced against cost-effectiveness considerations which have not been explicitly summarised.

While it was outside the scope of the present review to comprehensively summarise outcomes by specific therapeutic frameworks, it was noted that seven of the eight positive studies for distress used either SEGT or CBT [31, 33, 37, 38, 40, 45, 48]. Whilst the study combining CBT with SEGT reported only one significant result, improved helplessness/hopelessness [32], it should be noted that this RCT used an active treatment (self-help CBT) as the comparator. While it is possible that this self-help (low-intensity) CBT comparator provided equivalent benefit to more intensive psychological treatments, this was not tested, as the trial was not a non-inferiority study; head-to-head trials comparing efficacy across treatment-modalities could be usefully examined in future research. While lowintensity interventions did not have a sufficient evidence-base to recommend dissemination from the current review, this may be due to the fact that three of the four included lowintensity RCTs did not test established therapeutic frameworks. Indeed, the only lowintensity intervention testing an established framework (CBT) obtained more significant results than the other three low-intensity interventions that trialled other therapeutic frameworks [37].

While group programs clearly had the strongest empirical base for their use, it is notable that no new group-based RCTs have emerged since the last Cochrane Review on this topic. A number of reasons likely exist for this, including reduced research funding, general debate over the efficacy and acceptability of psychological interventions for cancer patients, and 
hence the value of further research on such interventions (e.g. [54, 55]). However, it is important to consider that the burden of participation and high cost/resource use are significant barriers to uptake of group interventions. Indeed, this study highlighted that groups required the highest levels of commitment, with an average of 60 hours of participation, nearly 10 times the commitment required for individual therapy and 15 times greater than low-intensity interventions. Overall, as intervention duration increased, uptake and adherence decreased. Disparities between those who took up interventions and those who declined were also discernible from this review: across the included studies that reported demographic characteristics, there was a general pattern for young, married/partnered, Caucasian, English-speaking women to be over-represented. Eligibility criteria for most studies also required women to live within close proximity of the Cancer Centre where the studies were being conducted (for face-to-face interventions), therefore reducing the likelihood of rural / regional women participating. Thus, given the high level of commitment required for group therapy, restrictions in uptake on rural/regional women, low adherence rates for individual therapies and higher adherence rates for low-intensity therapies found in this review, one way in which to address the need for accessible low-burden interventions may be to explore low-intensity interventions based on established therapeutic frameworks, such as SEGT, CBT or Acceptance and Commitment Therapy (ACT). Moreover, given the success of web-based interventions as a specific example of low-intensity intervention in treating distress related to a range of chronic physical health conditions [17], online interventions based on therapeutic frameworks may be the most obvious choice in balancing the need for efficacy with uptake and adherence in interventions available to women with MBC. This could also offer the benefit of providing online social support, as many online programs incorporate forums and can be run in group formats.

\section{Methodological quality}

None of the studies met all quality criteria, suggesting need for improvements in the methodological quality of research design and reporting. This does not imply that the studies were of poor quality; rather, that reporting was not transparent and many criteria could not be determined from the available information. It should be noted that over the 35-year timeframe that these studies were conducted, the reporting criteria required by journals have markedly changed, with methods developed to improve the quality of reporting of trials such as the CONSORT statement and checklist [56]. The substantial heterogeneity of research design, analytic strategy, comparator groups, methodological quality, sample sizes, and outcome measures utilised further increases the challenges arising when evaluating efficacy. For example, only seven of the 15 included RCTs specified a primary outcome; even when stated, these primary outcomes varied markedly across survival and psychosocial outcomes so it was not possible to summarise efficacy by primary outcomes. Methods for improving the quality of design could therefore include standardising the range of outcome measures utilised, specifying and powering to a primary outcome, and using treatment-as-usual or appropriate placebo-controls rather than waitlist or active-treatment comparators. As prior reviews have noted, waitlist designs are problematic as control-participants anticipate future therapeutic input and are therefore less likely to seek constructive action compared to other control-group types [57]. While active-comparators are methodologically strong, they are generally only 
adopted in settings where strong evidence-based gold-standard treatments exist and noninferiority designs are appropriate. Given the overall paucity of psychological intervention trials in MBC, it is premature to be testing non-inferiority, and efforts should instead be directed to building the evidence-base for psychological treatments. Two of the included studies utilised an active comparator [32,37]. Finally, ensuring that sample sizes are sufficient to meet power requirements for statistical analysis would greatly benefit this area, as only $40 \%$ of included RCTs were adequately powered.

\section{Limitations and future directions}

The current review has several limitations. First, the effect sizes of outcomes were not summarised, as these data were not routinely or consistently reported across trials. Second, studies often included multiple follow-up assessment points, however these points differed immensely between studies, or assessments were aggregated into an average/composite follow-up score making it difficult to summarise outcomes by time-periods. While all available data was extracted, this review elected to summarise the overall impact on psychosocial domains (that is, whether the intervention led to a significant group difference at any of the assessments), rather than differentiating the short- versus long-term impact, or changes over time within outcomes. However, for many of the included studies, psychosocial effects were short-term / not sustained or required a lengthy course of psychological treatment to obtain effects (e.g., a full year of SEGT to obtain statistically significant group differences). Third, in addition to reporting requirements changing over the 35-year period over which included studies were conducted, the oncology landscape for treating metastatic breast cancer (including average survival time, side effects, and resulting quality of life) has also changed significantly, with the advent of new systemic treatments [2]. Therefore any conclusions made when comparing psychological interventions delivered across this broad time-frame should be made with caution. Likewise, the discipline of psycho-oncology particularly our understanding of the mechanisms through which psychological interventions lead to change - has also matured over that time period. While SEGT and CBT remain the gold-standard treatments, more emphasis in recent times has been placed on the role of perseverative thinking (worry and rumination), attentional biases, and metacognitive processes as maintenance factors for psychological morbidity [58]. None of the included studies have examined interventions that specifically target these factors, such as the 'thirdwave' psychological intervention strategies (metacognitive or mindfulness-based therapies), despite a recent Breast Cancer Gap Analysis identifying this as a priority for research [59]. Given the promising evidence emerging for these new intervention types in non-metastatic populations [60] and in metastatic/advanced disease populations that are not breast-cancer specific [61-63], this is a useful avenue for future research. Finally, it should be noted that grey literature was excluded from this review, as there is a high discordance in the abstractto-publication rates and quality; grey literature often only reports preliminary results that are unrepresentative of final observations once all data is collected and analysed [64], with results changing in up to $42 \%$ of the abstract-to-publication comparisons [65]. Given this, only final fully-published (and data-extractable) peer-reviewed articles were eligible for inclusion. 


\section{Summary}

In summary, this review confirms the paucity of research addressing the unmet psychological needs of women with $\mathrm{MBC}$, and highlights the challenge of balancing efficacious treatments against accessibility, reduced burden, and increased acceptability. There is a lack of research evidence on efficacy of interventions in women of diverse ethnic background, socioeconomic status, and broader age-ranges. It is paramount to ensure interventions reach, and meet the needs of, a broad range of women with MBC, while simultaneously providing evidencebased benefits. While psychological therapies had consistent support for their use in improving distress, aspects of coping, and pain, when separated by treatment modality this evidence is only applicable to group-delivered programs at this stage; the evidence-base is insufficient for individual therapies and low-intensity interventions. More research is therefore required on how to deliver evidence-based interventions in an accessible lowburden format.

This article is protected by copyright. All rights reserved. 


\section{Acknowledgements}

Dr Beatty is funded from a NHMRC project grant (GNT 1042942); Prof Girgis is funded from a Cancer Institute NSW grant. The authors would like to thank Dr Addie Wootten for contributing to the design of this systematic review.

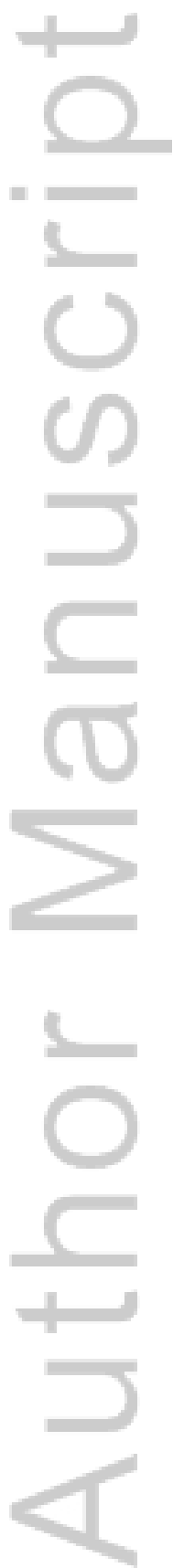

This article is protected by copyright. All rights reserved. 


\section{References}

1. Berman, A.T., et al., Incidence and patterns of distant metastases for patients with early-stage breast cancer after breast conservation treatment. Clinical Breast Cancer, 2013. 13(2): p. 88-94.

2. Tevaarwerk, A.J., et al., Survival in patients with metastatic recurrent breast cancer after adjuvant chemotherapy. Cancer, 2013. 119(6): p. 1140-1148.

3. Mosher, C.E. and K.N. Duhamel, An examination of distress, sleep, and fatigue in metastatic breast cancer patients. Psycho-Oncology, 2012. 21(1): p. 100-7.

4. Giese-Davis, J., et al., Decrease in depression symptoms is associated with longer survival in patients with metastatic breast cancer: a secondary analysis. Journal of Clinical Oncology, 2011. 29(4): p. 413-420.

5. Palesh, O.G., et al., A longitudinal study of depression, pain, and stress as predictors of sleep disturbance among women with metastatic breast cancer. Biological Psychology, 2007. 75(1): p. 37-44.

6. DiMatteo, M., H.S. Lepper, and T.W. Croghan, Depression is a risk factor for noncompliance with medical treatment: Meta-analysis of the effects of anxiety and depression on patient adherence. Archives of Internal Medicine, 2000. 160(14): p. 2101-2107.

7. Jassim, G.A., et al., Psychological interventions for women with non-metastatic breast cancer. Cochrane Database of Systematic Reviews, 2015. 5: p. CD008729.

8. Mustafa, M., et al., Psychological interventions for women with metastatic breast cancer. Cochrane Database of Systematic Reviews, 2013. 6: p. CD004253.

9. Beatty, L., B. Koczwara, and T. Wade, 'Cancer Coping Online': A pilot trial of a selfguided CBT internet intervention for cancer-related distress. Electronic Journal of Applied Psychology., 2011. 7(2): p. 17-25.

10. Beatty, L., B. Koczwara, and T.D. Wade, Evaluating the efficacy of a self-guided web-based CBT intervention for reducing cancer-distress: A pilot randomised controlled trial. Supportive Care in Cancer, 2016. 24(3): p. 1043-1051.

11. Carpenter, K.M., et al., An online stress management workbook for breast cancer. Journal of Behavioral Medicine, 2012.

12. Duffecy, J., et al., Project onward: an innovative e-health intervention for cancer survivors. Psycho Oncology, 2013. 22: p. 947-951.

13. Owen, J.E., et al., Randomized pilot of a self-guided internet coping group for women with early-stage breast cancer. Annals of Behavioral Medicine, 2005. 30(1): p. 54-64.

14. Scott, K. and L. Beatty, A feasibility study of a self-guided CBT internet intervention for cancer carers. Australian Journal of Primary Health, 2013. 19: p. 270-274.

15. van den Berg, S.W., et al., BREATH: Web-Based Self-Management for Psychological Adjustment After Primary Breast Cancer-Results of a Multicenter Randomized Controlled Trial. Journal Of Clinical Oncology: Official Journal Of The American Society Of Clinical Oncology, 2015.

16. Wootten, A.C., et al., Preliminary Results of a Randomised Controlled Trial of an Online Psychological Intervention to Reduce Distress in Men Treated for Localised Prostate Cancer. European Urology, (0). 
17. Beatty, L. and S. Lambert, A systematic review of internet-based self-help therapeutic interventions to improve distress and disease-control among adults with chronic health conditions. Clinical Psychology Review, 2013. 33: p. 609-622.

18. Willems, R.A., et al., Short-term effectiveness of a web-based tailored intervention for cancer survivors on quality of life, anxiety, depression, and fatigue: randomized controlled trial. Psycho-Oncology, 2016: p. n/a-n/a.

19. Spence, D., L. Morstyn, and K. Wells, The support and information needs of women with secondary breast cancer. 2015: Breast Cancer Network Australia.

20. Bennett-Levy, J., et al., eds. Oxford Guide to Low Intensity CBT Interventions. 2010, Oxford University Press: New York.

21. Rodgers, M., et al., The clinical effectiveness and cost-effectiveness of low-intensity - psychological interventions for the secondary prevention of relapse after depression: a systematic review. Health Technol Assess, 2012. 16(28): p. 1-130.

22. Brebach, R., et al., Psychological intervention targeting distress for cancer patients: a meta-analytic study investigating uptake and adherence. . Psycho-oncology, (2016). 25(8): p. 882-890.

23. Gerahty, A.W., A.M. Wood, and M.E. Hyland, Attrition from self-directed interventions: Investigating the relationship between psychological predictors, intervention content and dropout from a body dissatisfaction intervention. . Social Science and Medicine, 2010. 71(1 ): p. 30-37.

24. Moher, D., et al., Preferred Reporting Items for Systematic Reviews and MetaAnalyses: The PRISMA Statement. PLoS Med, 2009. 6(7): p. e1000097.

25. Higgins, J.P.T. and S. Green, Cochrane Handbook for Systematic Reviews of Interventions Version 5.0.1. [updated September 2008]. 2008, (http://www.cochranehandbook.org)/ The Cochrane Collaboration, 2008.

26. Chambless, D.L. and S.D. Hollon, Defining empirically supported therapies. Journal of Consulting \& Clinical Psychology, 1998. 66(1): p. 7-18.

27. Aranda, S., et al., Meeting the support and information needs of women with advanced breast cancer: a randomised controlled trial. British Journal of Cancer, 2006. 95(6): p. 667-73.

28. Arathuzik, D., Effects of cognitive-behavioral strategies on pain in cancer patients. Cancer Nursing, 1994. 17(3): p. 207-14.

29. Bordeleau, L., et al., Quality of life in a randomized trial of group psychosocial support in metastatic breast cancer: overall effects of the intervention and an exploration of missing data. Journal of Clinical Oncology, 2003. 21(10): p. 1944-51.

30. Butler, L.D., et al., Effects of supportive-expressive group therapy on pain in women with metastatic breast cancer. Health Psychology, 2009. 28(5): p. 579-87.

31. Classen, C., et al., Supportive-expressive group therapy and distress in patients with metastatic breast cancer: a randomized clinical intervention trial. Archives of General Psychiatry, 2001. 58(5): p. 494-501.

32. Cunningham, A.J., et al., A randomized controlled trial of the effects of group psychological therapy on survival in women with metastatic breast cancer. PsychoOncology, 1998. 7(6): p. 508-17. 
33. Edelman, S., D.R. Bell, and A.D. Kidman, A group cognitive behaviour therapy programme with metastatic breast cancer patients. Psycho-Oncology, 1999. 8(4): p. 295-305.

34. Edelman, S., et al., Effects of group CBT on the survival time of patients with metastatic breast cancer. Psycho-Oncology, 1999. 8(6): p. 474-81.

35. Edmonds, C.V., G.A. Lockwood, and A.J. Cunningham, Psychological response to long term group therapy: A randomized trial with metastatic breast cancer patients. Psycho-Oncology, 1999. 8(1): p. 74-91.

36. Giese-Davis, J., et al., Change in emotion-regulation strategy for women with metastatic breast cancer following supportive-expressive group therapy. Journal of Consulting \& Clinical Psychology, 2002. 70(4): p. 916-25.

37. = Given, C.W., et al., Managing symptoms among patients with breast cancer during chemotherapy: results of a two-arm behavioral trial. Journal of Clinical Oncology, 2008. 26(36): p. 5855-5862.

38. Goodwin, P.J., et al., The effect of group psychosocial support on survival in metastatic breast cancer. New England Journal of Medicine, 2001. 345(24): p. 171926.

39. Gotay, C.C., et al., Impact of a peer-delivered telephone intervention for women experiencing a breast cancer recurrence. Journal of Clinical Oncology, 2007. 25(15): p. 2093-9.

40. Kissane, D.W., et al., Supportive-expressive group therapy for women with metastatic breast cancer: Survival and psychosocial outcome from a randomized controlled trial. Psycho-Oncology, 2007. 16(4): p. 277-286.

41. Laidlaw, T., et al., Quality of life and mood changes in metastatic breast cancer after training in self-hypnosis or Johrei: a short report [corrected] [published erratum appears in CONTEMP HYPNOSIS 2005;22(3):169]. Contemporary Hypnosis (John Wiley \& Sons, Inc.), 2005. 22(2): p. 84-93.

42. Low, C.A., et al., A randomized controlled trial of emotionally expressive writing for women with metastatic breast cancer. Health Psychology, 2010. 29(4): p. 460-466.

43. Mosher, C.E., et al., Randomised trial of expressive writing for distressed metastatic breast cancer patients. Psychology \& Health, 2012. 27(1): p. 88-100.

44. Scholten, C., et al., Difference in patient's acceptance of early versus late initiation of psychosocial support in breast cancer. Supportive Care in Cancer, 2001. 9(6): p. 45964.

45. Spiegel, D., J.R. Bloom, and I. Yalom, Group support for patients with metastatic cancer. A randomized prospective outcome study. Archives of General Psychiatry, 1981. 38(5): p. 527-533.

46. Spiegel, D., et al., Effects of supportive-expressive group therapy on survival of patients with metastatic breast cancer: A randomized prospective trial. Cancer, 2007. 110(5): p. 1130-1138.

47. Spiegel, D., et al., Effect of psychosocial treatment on survival of patients with metastatic breast cancer. The Lancet, 1989. 334(8668): p. 888-891. 
48. Savard, J., et al., Randomized clinical trial on cognitive therapy for depression in women with metastatic breast cancer: Psychological and immunological effects. Palliative \& Supportive Care, 2006. 4(03): p. 219-237.

49. Spiegel, D. and J.R. Bloom, Group therapy and hypnosis reduce metastatic breast carcinoma pain. Psychosom Med, 1983. 45(4): p. 333-9.

50. Pinquart, M. and P.R. Duberstein, Depression and cancer mortality: a meta-analysis. Psychol Med, 2010. 40(11): p. 1797-810.

51. Le, C.P., et al., Chronic stress in mice remodels lymph vasculature to promote tumour cell dissemination. Nat Commun, 2016. 7.

52. Aizer, A.A., et al., Marital Status and Survival in Patients With Cancer. Journal of Clinical Oncology, 2013. 31(31): p. 3869-3876.

53. = Mausbach, B.T., R.B. Schwab, and S.A. Irwin, Depression as a predictor of adherence to adjuvant endocrine therapy (AET) in women with breast cancer: a systematic review and meta-analysis. Breast Cancer Research and Treatment, 2015. 152(2): p. 239-246.

54. Lepore, S.J. and J.C. Coyne, Psychological interventions for distress in cancer patients: A review of reviews. Annals of Behvioral Medicine, 2006. 32(2): p. 85-92.

55. Manne, S.L. and M.A. Andrykowski, Seeing the forest for the trees: A rebuttal. Annals of Behavioral Medicine, 2006. 32(2): p. 111-114.

56. Boutron, I., et al., Methods and Processes of the CONSORT Group: Example of an Extension for Trials Assessing Nonpharmacologic Treatments. Annals of Internal Medicine, 2008(148): p. W60-W66.

57. Cuijpers, P., A. van Straten, and G. Andersson, Internet-administered cognitive behavior therapy for health problems: a systematic review. Journal of Behavioral Medicine, 2008. 31(2): p. 169-177.

58. Ehring, T. and E.R. Watkins, Repetitive Negative Thinking as a Transdiagnostic Process. International Journal of Cognitive Therapy, 2008. 1(3): p. 192-205.

59. Eccles, S.A., et al., Critical research gaps and translational priorities for the successful prevention and treatment of breast cancer. Breast Cancer Research, 2013. 15(5).

60. Hulbert-Williams, N.J., L. Storey, and K.G. Wilson, Psychological interventions for patients with cancer: psychological flexibility and the potential utility of Acceptance and Commitment Therapy. European Journal of Cancer Care, 2015. 24(1): p. 15-27.

61. Breitbart, W., et al., Meaning-centred group psychotherapy for pateitns with advanced cancer: a pilot randomized controlled trial. Psycho-Oncology, 2010. 19(1): p. 21-28.

62. Chocinov, H.M., et al., Effect of dignity therapy on distress and end-of-life experience in terminally ill patients: a randomised controlled trial. . Lancet Oncology, 2011. 12(8): p. 753-762.

63. Lo, C., et al., Managing Cancer and Living Meaningfully (CALM): Phase 2 trial of a brief individual psychotherapy for patients with advanced cancer. Palliative Medicine, 2014. 28(3): p. 234-242.

64. Toma, M., et al., TRansition from meeting abstract to full-length journal article for randomized controlled trials. JAMA, 2006. 295(11): p. 1281-1287. 
65. Tam, V.C. and S.J. Hotte, Consistency of Phase III Clinical Trial Abstracts Presented at an Annual Meeting of the American Society of Clinical Oncology Compared With Their Subsequent Full-Text Publications. Journal of Clinical Oncology, 2008. 26(13): p. 2205-2211.

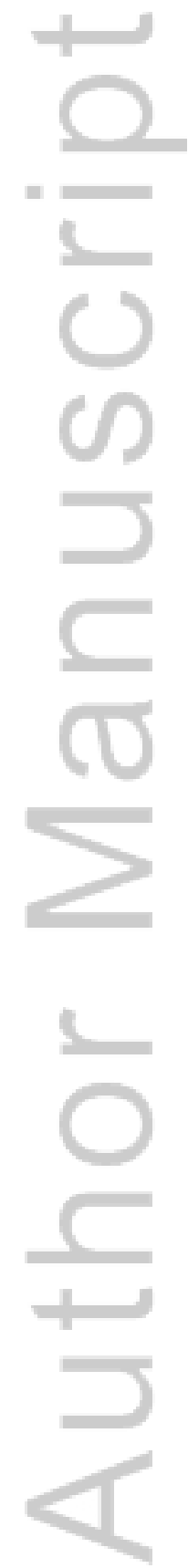

This article is protected by copyright. All rights reserved. 
Table 1. Outcome summary: number of positive vs negative trials for trial-outcomes, by treatment-delivery modality

\begin{tabular}{|c|c|c|c|c|c|c|c|c|c|c|c|}
\hline \multirow{2}{*}{ Outcome } & \multirow{2}{*}{$\begin{array}{c}\text { Number } \\
\text { of } \\
\text { RCTs }\end{array}$} & \multicolumn{3}{|c|}{ Group } & \multicolumn{3}{|c|}{ Individual } & \multicolumn{3}{|c|}{ Low Intensity } & \multirow{2}{*}{$\begin{array}{c}\text { RCTs } \\
\text { Pooled } \\
\text { Conclusior }\end{array}$} \\
\hline & & Positive & Negative & Conclusion & Positive & Negative & Conclusion & Positive & Negative & Conclusion & \\
\hline Survival & 6 & 1 & 5 & No support & 0 & 0 & $n / a$ & 0 & 0 & $\mathrm{n} / \mathrm{a}$ & No suppor \\
\hline Distress & 13 & 6 & 1 & Supported & 1 & 1 & Unclear & 1 & 3 & No support & Supported \\
\hline Coping & 5 & $4^{\mathrm{a}}$ & 0 & Supported & 0 & 1 & Unclear & 0 & 0 & $\mathrm{n} / \mathrm{a}$ & Supported \\
\hline Quality of Life & 6 & $1^{\mathrm{b}}$ & 2 & Unclear & 0 & 3 & No support & 0 & 0 & $\mathrm{n} / \mathrm{a}$ & No suppor \\
\hline Pain & 5 & 3 & 0 & Supported & 1 & 0 & Unclear & 0 & 1 & Unclear & Supported \\
\hline Fatigue & 5 & 1 & 1 & Unclear & 0 & 1 & Unclear & 0 & 2 & No support & No suppor \\
\hline Sleep & 4 & 0 & 0 & $\mathrm{n} / \mathrm{a}$ & 0 & 1 & Unclear & $2^{c}$ & 1 & Support & Unclear \\
\hline
\end{tabular}

${ }^{\text {a }}$ Supported for helplessness, restraint and 'maladaptive-coping' subscale; not for denial or defensive-repressive

${ }^{\mathrm{b}}$ Only for social functioning

${ }^{c}$ For one of these studies, benefits found only among those recently diagnosed [42].

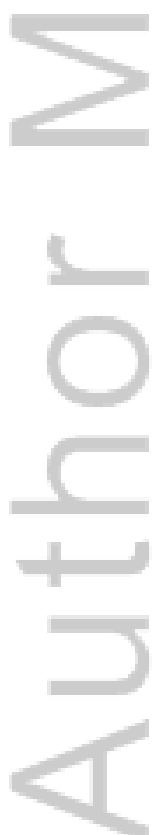

This article is protected by copyright. All rights reserved. 
Table 2. Trial context factors and intervention duration, by treatment delivery modality

\begin{tabular}{|c|c|c|c|c|}
\hline Outcomes & Group (n=7) & Individual $(n=4)$ & Low Intensity $(n=4)$ & Pooled $(n=15)$ \\
\hline \multicolumn{5}{|l|}{ Trial Characteristics } \\
\hline Average SS per trial & 235) & $M=54.3$ & $M=129.5$ & M: 109 \\
\hline & $M=128$ & & & \\
\hline Age, years (n RCTs) & $51.60(\mathrm{~N}=6)$ & $56.29(\mathrm{~N}=3)$ & $54.73(\mathrm{~N}=4)$ & $53.08(\mathrm{~N}=13)$ \\
\hline $\begin{array}{l}\text { Years since MBC diagnosis ( } \\
\text { RCTs) }\end{array}$ & $2.50(\mathrm{~N}=4)$ & $0.75(\mathrm{~N}=3)$ & $2.64(\mathrm{~N}=3)$ & $1.96(\mathrm{~N}=10)$ \\
\hline Married (n RCTs) & $67.5 \%(\mathrm{~N}=6)$ & $66.3 \%(\mathrm{~N}=3)$ & $71.3 \%(\mathrm{~N}=3)$ & $68.4 \%(\mathrm{~N}=12)$ \\
\hline Tertiary educated (n RCTs) & $47.3 \%(\mathrm{~N}=2)$ & $58.5 \%(\mathrm{~N}=3)$ & $80.9 \%(\mathrm{~N}=3)$ & $62.2 \%(\mathrm{~N}=8)$ \\
\hline Employed (n RCTs) & $36 \%(\mathrm{~N}=2)$ & $31.3 \%(\mathrm{~N}=2)$ & $28.5 \%(\mathrm{~N}=2)$ & $31.9 \%(\mathrm{~N}=6)$ \\
\hline \multicolumn{5}{|l|}{ Intervention characteristics } \\
\hline Uptake (n RCTs; range) & $41.9 \%(\mathrm{~N}=6 ; 19-79 \%)$ & $60.5 \%(\mathrm{~N}=2 ; 60-61 \%)$ & $79.1 \%(\mathrm{~N}=2 ; 69-89 \%)$ & $53.02(\mathrm{~N}=10 ; 19-89 \%)$ \\
\hline Adherence (n RCTs; range) & $66.1 \%(\mathrm{~N}=7 ; 43-82 \%)$ & $62.6 \%(\mathrm{~N}=4 ; 40-100 \%)$ & $85.9 \%(\mathrm{~N}=4 ; 80-96 \% \%)$ & $69.3 \%(\mathrm{~N}=15,40-100 \%)$ \\
\hline Intervention average duration (range) & 60 hours $(16-78)$ & 6.83 hours $(2-16.5)^{\mathrm{a}}$ & 3.79 hours $(1.33-6.5)$ & 32.6 hours $(1.33-78)$ \\
\hline
\end{tabular}

This article is protected by copyright. All rights reserved. 


\section{University Library}

\section{- M M N E R VA A gateway to Melbourne's research publications}

Minerva Access is the Institutional Repository of The University of Melbourne

\section{Author/s:}

Beatty, L;Kemp, E;Butow, P;Girgis, A;Schofield, P;Turner, J;Hulbert-Williams, NJ;Levesque, $\mathrm{JV} ;$ Koczwara, B

Title:

A systematic review of psychotherapeutic interventions for women with metastatic breast cancer: Context matters

Date:

2018-01-01

Citation:

Beatty, L., Kemp, E., Butow, P., Girgis, A., Schofield, P., Turner, J., Hulbert-Williams, N. J., Levesque, J. V. \& Koczwara, B. (2018). A systematic review of psychotherapeutic interventions for women with metastatic breast cancer: Context matters. PSYCHOONCOLOGY, 27 (1), pp.34-42. https://doi.org/10.1002/pon.4445.

Persistent Link:

http://hdl.handle.net/11343/292912 